\title{
The Epithelial Cytokeratins 15 and 19 Gene Expression in Non-Segmental Vitiligo Patients under NB-UVB Phototherapy
}

\author{
A.I.El-taweel ${ }^{1}$, N.E.Sorour ${ }^{1}$, N.F.Elhussieni ${ }^{2}$, H.E.Elaal ${ }^{1}$
}

${ }^{1}$ Dermatology \& Andrology Dept., Faculty of Medicine, Benha Univ., Benha, Egypt

${ }^{2}$ Medical Biochemistry Dept., Faculty of Medicine, Benha Univ., Benha, Egypt

E-Mail:hanaaeid90@yahoo.com

\begin{abstract}
Cytokeratins 15 and 19 were first suggested as a specific stem cell marker, demonstrated their restricted expression in the bulge region of murine and human hair follicles. It was proved that they are valuable tools to monitor tissue homeostasis and the potential to self-renew.The aim of this work was to investigate the expression of CK15 and CK19 as possible stem cell markers in the skin of vitiligo patients during NB-UVB phototherapy repigmentation. The current study was a case-control study that was conducted on 40 participants. Fifty of them were patients with vitiligo, and they were located in group 1. Group 2 contained 20 age and sex matched healthy control participants. Approval of the Local Ethics Committee on Research involving human subjects of Benha Faculty of Medicine and written informed consents of participants were obtained before samples collection. Quantitative assessment of cytokeratins 15\& 19 gene expression in all biopsies by Real Time Polymerase Chain Reaction. the level of cytokeratins were found to be higher in the patients after they received NB-UVB than control and it was found a significant relation with the improvement of the disease.Cytokeratin $15 \& 19$ are valuable tools to monitor vitiligo improvement.
\end{abstract}

Keywords: Vitiligo, Cytokeratins, Stem Cells.

\section{Introduction}

About $1 \%$ of people worldwide are affected by vitiligo [1] Males and females are equally affected. About half show the disorder before age 20 and 95\% develop it before age 40 [2].

The cause is typically unknown. It is believed to be due to genetic susceptibility that is triggered by an environmental factor such that an autoimmune disease occurs. This results in destruction of skin pigment cells. A family history of the condition or other autoimmune diseases, such as hyperthyroidism, alopecia areata, and pernicious anemia were recognized as Risk factors [3] .

Up till now, no cures for vitiligo but several treatment options are available such as topical steroids, calcineurin inhibitors, phototherapy, laser and surgical treatment [3].

Replenishing the skin with new melanocytes is needed during the repigmentation process and is dependent on existence of stem cells [4]. Narrow band ultra violet radiation induces repigmentation in distinctive patterns such as follicular, marginal, and diffuse. Follicular pattern is the predominant, and comes from hair follicle melanocytes [5].

The hair follicle is a rich source of different subtypes of stem cells, which are known as hair follicle stem cells (HFSCs) [6] HFSCs are multipotent stem cells that are located in the lower permanent bulge and subbulge area of the hair follicle. In addition, dermal mesenchymal stem cells are found in the perifollicular connective tissue sheath and the dermal papilla [7] and melanocyte stem cells (MelSCs) that are responsible for repigmentation in vitiligo by dividing and migrating upward along the surface of the hair follicle to spread in a concentric pattern causing repigmentation8] These DOPA-negative "inactive" melanocytes (MelSCs) residing in the outer root sheath, and bulge areas of the hair follicle were also described several years ago in a landmark study by Staricco who claimed their activation when stimulated by ultraviolet radiation. This phototherapy-induced stimulation is now a well-established fact [9].

Cytokeratins (CK) belong to the family of intermediate filament proteins that are specifically expressed in epithelia. It has been suggested that specific cytokeratins are markers for stem cells activation [10].

\section{Subjects and methods}

The current study was a case-control study that was conducted on patients with vitiligo in Benha University Hospital. This study was conducted on 40 participants. Fifty of them were patients with vitiligo, and they were located in group 1 . Group 2 contained 20 control participants matching to the patients in age and sex. Approval of the Local Ethics Committee on Research involving human subjects of Benha Faculty of Medicine and written informed consents of participants were obtained before samples collection.

In group 1, the patients assessed with full history taking, general and dermatological examination with photographic documentation. The severity of the condition was assessed by VETI and VASI scores. Then skin biobsies were taken for laboratory investigations. Quantitative assessment of cytokeratin 15 and 19 gene expression by real time PCR.

Qualitative data were summarized in the form of frequency and percentage. Mean and SD were obtained for quantitative data, while categorical data were presented by number and percentage. One way analysis of variance (ANOVA) test, 
paired and independent sample t-tests were used for comparing means between groups. Tests used for association were Chi square (X2) or Mont-Carlo Exact test (MCET). P -value was adopted to be $<0.05$.

\section{Results}

The patients and control groups were matching regarding age $(33.6 \pm 12.6$ versus $33.9 \pm 12, \mathrm{p}=0.93)$ and gender (35\% male patients versus $40 \%$ male control, $\mathrm{p}=0.23$ ). The clinical findings of patients are shown in Table (1).

By studying the family history, out of 20 patients, only 7 patients (35\%) reported positive consanguinity. Also 7 patients $(35 \%)$ had a similar condition in their family.

During taking the medical history, no any studied patients had associated auto immune diseases. Out of the studied patients, only 2 patients (10\%) had diabetes mellitus and 3 patients $(15 \%)$ were hypertensive. 5 patients $(25 \%)$ reported intake of systemic drugs for their diseases.

As regarding to the duration of the disease, 14 patients $(70 \%)$ reported that they had vitiligo for less than 10 years, while the rest $(30 \%)$ suffered from the disease for 10 years or more.

By studying the disease stability among studied patients, 7 patients $(35 \%)$ reported new lesions in less than 2 months, $24 \%$ reported new lesions within 2 to 6 months and the rest of the patients $(41 \%)$ reported stable disease for more than 6 months.

There were several exacerbating factors for the disease among studied patients. 8 patients $(40 \%)$ had only one exacerbating factor for their disease, 8 patients $(40 \%)$ had several exacerbating factors while the rest $(20 \%)$ did not reported any factors. Stress was the commonest factor could exacerbate the disease in our studied patients $(76 \%)$ then trauma $(28 \%)$ then sun exposure $(16 \%)$ and lastly infections (4\%) Table( 2).

Out of 20 patients, only 5 patients (25\%) developed white hairs at the site of vitiligo while the rest $(75 \%)$ had normal pigmented hairs.

Cytokeratin 15\&19 levels were found to be higher among studied patients than the control group Table $(3 \& 4)$.

\section{Discussion}

As vitiligo is poly genetic disease, positive family history is one of the strong association with it. Positive consanguinity and family history of similar condition is reported by $35 \%$ of our patients. This agrees with Mohammed et al., [11] who reported that the range of family history in vitiligo varied from $6.25 \%$ up to $38 \%$. But this percentage is higher than that reported by Butt et al.,[12] who found positive family history in $22 \%$ of his study. The patients were considered to have a family history if they had one or more first to third degree relatives with this condition.
Vitiligo is widely accepted to be an autoimmune disease and may be associated with other autoimmune diseases as suggested by Amerson and Murphy [13] who reported that 20\% of their patients had at least one autoimmune disease. This disagree with the current study, as no reported autoimmune diseases among the patients due to exclusion of diabetes mellitus that reported by $10 \%$ of the studied patients, based on the fact that the autoimmune mechanism plays a minor role in in pathogenesis of the disease especially type II, as genetic, ethnic, environmental as well as nutritional factors play the main pathogenic roles [14].

By analysis of the disease characteristics among the patients, $70 \%$ reported early onset of the disease $(<10$ years, while the rest had late onset $(10$ years or more). This classification agrees with Teulings et a.,1 [15.]

Through studying the activity of the disease among the patients, it is found that $35 \%$ reported new lesions in less than 2 months (active or unstable disease), 24\% reported new lesions within 2 to 6 months and the rest of the patients $(41 \%)$ reported stable disease for more than 6 months. This agrees with Lahiri [16] who suggested that the minimum period for clinical stability required for surgery ranges from 4 months to 2 years and unstable disease manifested by appearance of new lesions within 6 weeks.

There are several exacerbating factors for the vitiligo, among the studied patients $40 \%$ had only one exacerbating factor for their disease, $40 \%$ had several exacerbating factors while the rest $(20 \%)$ did not reported any factors. Stress was the commonest factor could exacerbate the disease in our studied patients $(76 \%)$ then trauma $(28 \%)$ then sun exposure (16\%) and lastly infections (4\%). These results are comparable to the results of Jeon et al., [17] who reported that emotional stress $(55.4 \%)$ was the most frequent provoking factor, followed by sunburn $(28.8 \%)$, mechanical factors (19.2\%), and chemical factors (16.4\%). Also, it agrees with Silverberg and Silverberg [18] who performed a questionnaire-based study of 1541 adults with vitiligo and found that $56.6 \%$ of subjects reported at least 1 death or stressor within 2 years before vitiligo onset.

Regarding the color of hair in vitiligenous lesions, $25 \%$ of the patients developed white hairs at the site of vitiligo while the rest $(75 \%)$ had normal pigmented hairs. This agrees with Kim et al. [19] who reported that leukotrichia occurs in 8.9- $45 \%$ of vitiligo cases. But this disagrees with Elmasry et al. [20] who found, leukotrichia was found in $46.5 \%$ of their studied patients. This difference may be explained by different age, duration and activity among the patients.

Through measuring the level of cytokeratins 15 and 19 among the patients in comparison with control, we found that cytokeratins are expressed in 
normal control but with low level, and this agrees with Zhou et al., [21] who studied these CKs expression in adnexal neoplasm in comparison with normal subjects. When CKs were measured in the patients after they received NB-UVB for $4^{\text {th }}$ months, they are found higher than control and this agrees with Goldstein et al. [22] who reported the effect of NB-UVB on proliferation and differentiation of Melscs.

Table (1) Socio demographic data of participants

\begin{tabular}{|c|c|c|c|c|c|c|}
\hline \multicolumn{2}{|c|}{ Variable } & \multicolumn{2}{|c|}{$\begin{array}{c}\text { Vitiligo patients } \\
(\mathbf{N}=\mathbf{2 0})\end{array}$} & \multicolumn{2}{|c|}{$\begin{array}{c}\text { Controls } \\
(\mathrm{N}=20)\end{array}$} & \multirow{2}{*}{$\mathbf{P}$} \\
\hline & & No. & $\%$ & No. & $\%$ & \\
\hline \multirow{2}{*}{ Sex } & Male & 7 & 35 & 8 & 40 & \multirow{4}{*}{$\begin{array}{l}0.23 \\
(\mathrm{NS})\end{array}$} \\
\hline & Female & 13 & 65 & 12 & 60 & \\
\hline \multirow{2}{*}{ Age (ys) } & Mean \pm SD & $33.6 \pm 12.6$ & $33.9 \pm 12.5$ & St."t"= & 0.93 & \\
\hline & (Range) & $(13-52)$ & $(13-50)$ & 0.082 & (NS) & \\
\hline
\end{tabular}

Table (2) Types of exacerbating factors for the disease among studied patients.

\begin{tabular}{lccc}
\hline Variable & & No. $(\mathbf{N}=\mathbf{2 0})$ & $\mathbf{\%}(\mathbf{1 0 0 \%})$ \\
\hline Exacerbating factors & Sun exposure & 5 & 25 \\
& Trauma & 7 & 35 \\
& Psychogenic & 17 & 85 \\
& Infection & 1 & 5 \\
& Drugs & 0 & 0.0 \\
& Chemicals & 0 & 0.0 \\
\hline
\end{tabular}

Table (3) C.K 15 among patients versus control.

\begin{tabular}{lccc}
\hline CK 15 & Patient 4m. & Patient 6m. & Control \\
\hline Range & $3.61-4.3$ & $4.21-4.92$ & $2.98-3.68$ \\
Mean \pm SD & $5.12 \pm 0.13$ & $5.34 \pm 0.15$ & $4.12 \pm 0.12$ \\
P. value & & $0.001^{*}$ & \\
4m \& 6m & $4 \mathrm{~m} \&$ Control & & $6 \mathrm{~m}$ \& Control \\
\hline $\mathbf{0 . 0 0 1 *}$ & $0.001^{*}$ & & $0.001^{*}$ \\
\hline
\end{tabular}

Table (4) C.K 19 among patients versus control

\begin{tabular}{lccc}
\hline CK 19 & Patient 4m. & Patient 6m. & Control \\
\hline Range & $3.6-4.31$ & $4.15-4.68$ & $3.00-3.69$ \\
Mean + SD & $4.2 \pm 0.12$ & $5.35 \pm 0.14$ & $4.00 \pm 0.12$ \\
P. value & & $0.001^{*}$ & \\
4m \& 6m & $4 \mathrm{~m} \&$ Control & & $6 \mathrm{~m}$ \& Control \\
\hline 0.001* & $0.001^{*}$ & & $0.001^{*}$ \\
\hline
\end{tabular}

\section{Conclusion}

Ctokeratins $15 \& 19$ can be used as markers for melanocyte stem cells proliferation in vitiligo.

\section{References}

[1] M. Whitton, M. Pinart and J. Batchelor: Evidencebased management of vitiligo: summary of a Cochrane systematic review. Br j dermatol, Vol. 174 (5), PP. 962-9, 2016.

[2] R. Yaghoobi, M. Omidian and N. Bagherani: Vitiligo: a review of the published work. J Dermatol, Vol. 38, PP. 419-31, 2011.

[3] K. Ezzedine, V. Eleftheriadou, M. Whitton and N. van Geel: Vitiligo. Lancet (London, England). Vol.386, PP. 74-84, 2015.
[4] S. Awad: New population of amelanotic spindle cells are clearly demonstrated in vitiliginous skin after ultraviolet radiation. J Eur Acad Dermatol Venereol, Vol. 28, PP. 1811-5, 2014.

[5] V. Lepe, B. Moncada, J. Castanedo-Cazares, M. Torres-Alvarez, C. Ortiz and A. TorresRubalcava: A double-blind randomized trial of $0.1 \%$ tacrolimus vs $0.05 \%$ clobetasol for the treatment of childhood vitiligo. Arch Dermatol. Vol.139, PP.581-5, 2003.

[6] H. Yu, S. Kumar and A. Kossenkov: Stem cells with neural crest characteristics derived from the bulge region of cultured human hair follicles. $\mathbf{J}$ Invest Dermatol, Vol.130, PP. 1227-36, 2010. 
[7] K. Sellheyer and D. Krahl: Skin mesenchymal stem cells: prospects for clinical dermatology. J Am Acad Dermatol, Vol. 63, PP. 859-65, 2010.

[8] E. Nishimura, S. Jordan and H. Oshima: Dominant role of the niche in melanocyte stem-cell fate determination. Nature, Vol.416, PP. 854-60, 2002.

[9] R. Staricco: Amelanotic melanocytes in the outer sheath of the human hair follicle. J Invest Dermatol, Vol. 33, PP.295-7, 1959.

[10] O. Abbas and M. Mahalingam: Epidermal stem cells: practical perspectives and potential uses. $\mathrm{Br}$ J Dermatol, Vol.161, PP. 228-236, 2009.

[11] G. Mohammed, A. Gomaa and M. Al-Dhubaibi: Highlights in pathogenesis of vitiligo. World. J Clin Cases, Vol.3, PP.221, 2015.

[12] G. Butt, F. Altaf, U. Wazir and S. Pal: Familial frequency of vitiligo and its association with autoimmune disorders. J Pak Assoc Derma, Vol.15, PP. 101-104, 2015.

[13] E. Amerson and E. Murphy: Comorbid autoimmune diseases in patients with vitiligo: A cross-sectional study. J Am Acad Dermatol, Vol.75, PP. 231, 2016.

[14] J. Skyler, G. Bakris, E. Bonifacio, T. Darsow, R. Eckel and L. Groop: Differentiation of Diabetes by Pathophysiology, Natural History, and Prognosis. Diabetes, Vol. 66(2), PP.241-255, 2016.

[15] H. Teulings, E. Ceylan, M. Overkamp, C. Vrijman, Bos $\mathbf{J}$ and $\mathrm{T}$. Nijsten,: Non segmental vitiligo disease duration and female sex are associated with comorbidity and disease extent: a retrospective analysis in 1307 patients aged $\geq 50$ years. Br J Dermatol, Vol.175 (4), PP.821-824, 2016.
[16] K. Lahiri: Stability in vitiligo? What's that?. Journal of Cutaneous and Aesthetic Surgery, Vol.2 (1), PP.38, 2009.

[17] I. Jeon, C. Park, M. Lee, D. Lee, H. Kang and S. Hann: A Multicenter Collaborative Study by the Korean Society of Vitiligo about Patients' Occupations and the Provoking Factors of Vitiligo. Ann Dermatol, Vol. 26(3), PP.349, 2014.

[18] J. Silverberg and N. Silverberg: Vitiligo disease triggers: Psychological stressors preceding the onset of disease. Cutis, Vol.95, PP.255-262, 2015.

[19] M. Kim, E. Cho, E. Park, K. Kim and K. Kim: Effect of excimer laser treatment on vitiliginous areas with leukotrichia after confirmation by dermoscopy. Int J Dermatol, Vol.55, PP.886-92, 2016.

[20] M. Elmasry, R. Mogawer and W. Mostafa: New insights into leukotrichia in nonsegmental vitiligo: A cross-sectional study. Indian J Dermatol Venereol Leprol,Vol.85, PP.374, 2019.

[21] N. Goldstein, M. Koster, L. Hoaglin, N. Spoelstra, K. Kechris and S. Robinson: Narrow Band Ultraviolet B Treatment for Human Vitiligo Is Associated with Proliferation, Migration, and Differentiation of Melanocyte Precursors. J Investig Dermatol, Vol.135, PP.2068-2076, 2015.

[22] X. Zhou, G. Li, D. Wang, X. Sun and X. Li: Cytokeratin expression in epidermal stem cells in skin adnexal tumors. Oncol Lett, Vol.17, PP.927932, 2019. 